\title{
The Pros and Cons of Computer-Aided Surgery for Segmental Mandibular Reconstruction after Oncological Surgery
}

\author{
Hyun Ho Han ${ }^{1}$, \\ Hak Young Kim², \\ Jun Yong Lee ${ }^{2}$ \\ ${ }^{1}$ Department of Plastic Surgery, Asan \\ Medical Center, University of Ulsan College of \\ Medicine, Seoul; \\ ${ }^{2}$ Department of Plastic and Reconstructive \\ Surgery, College of Medicine, The Catholic \\ University of Korea, Seoul, Korea
}

No potential conflict of interest relevant to this article was reported.

\begin{abstract}
Computer-aided surgery (CAS) started being used for head and neck reconstruction in the late 2000s. Its use represented a paradigm shift, changing the concept of head and neck reconstruction as well as mandible reconstruction. Reconstruction using CAS proceeds through 4 phases: planning, modeling, surgery, and evaluation. Thus, it can overcome a number of trial-and-error issues which may occur in the operative field and reduce surgical time. However, if it is used for oncologic surgery, it is difficult to evaluate tumor margins during tumor surgery, thereby restricting pre-surgical planning. Therefore, it is dangerous to predetermine the resection margins during the presurgical phase and the variability of the resection margins must be taken into consideration. However, it allows for the preparation of a prebending plate and planning of an osteotomy site before an operation, which are of great help. If the current problems are resolved, its applications can be greatly extended.
\end{abstract}

Keywords: Computer-aided surgery / Mandible / Mandibular reconstruction / Resection margin / Microsurgical free flaps

\section{INTRODUCTION}

Mandible reconstruction after oncological resection is a challenging task. In particular, it is very challenging as it involves consideration of occlusion, bone-to-bone contact, dental alignment, and aesthetic contours in the intraoperative field after segmental resection [1,2]. The best current method of reconstructing segmental mandibular defects after ablative surgery for head and neck cancer is the use of an osteocutaneous free fibula flap [3-6].

In the past, the use of an osteocutaneous free fibula flap during mandibular reconstruction depended on the surgeon's skill, the surgeon's judgment in the moment, and trial-and-error in the op-

Correspondence: Jun Yong Lee

Department of Plastic and Reconstructive Surgery, Incheon St. Mary's Hospital, College of Medicine, The Catholic University of Korea, 56 Dongsu-ro, Bupyeong-gu, Incheon 21431, Korea

E-mail: ps.jylee@catholic.ac.kr

Received August 9, 2017 / Revised September 13, 2017 / Accepted September 13, 2017 erating room; for surgeons, it was one of the most difficult reconstructive surgeries because of its steep learning curve $[7,8]$.

To overcome this problem, since the late 2000s, computer-aided surgery (CAS) has been introduced and applied to many complex craniomaxillofacial reconstruction procedures, especially reconstruction of semgemtal mandibular defects that occur after oncologic ablative surgery $[1,7-13]$. Therefore, this change, which can be thought of as a paradigm shift, served as a turning point for head and neck reconstruction, just as smartphones have changed modern lifestyles [9].

CAS technology, including virtual surgical planning, computer-aided design (CAD), computer-aided modeling (CAM), rapid prototyping, intraoperative navigation, and so on, greatly aids in the planning of reconstructive surgery [14].

For mandibular reconstructive oncological surgery, it is critical to secure a sufficient cancer-free margin of bone and soft tissue in the intraoperative field as well as to consider occlusion, bone-to- 
bone contact, dental alignment, and aesthetic contours. However, for these reasons, there are obvious differences between reconstruction after the removal of a non-malignant tumor and the application of CAS in post-traumatic reconstruction and in cases of congenital reconstruction, etc.

Therefore, this paper will review the methods of reconstruction using CAS for oncological surgery of the mandible and address the problems that currently remain, as well as discuss directions for the future.

\section{SURGICAL TECHNIQUE AND PLANNING}

Like other complex craniofacial reconstruction techniques, reconstruction using CAS in the mandible proceeds through 4 phases: planning, modeling, surgery, and evaluation $[9,10,15,16]$.

The first phase is planning. This phase includes conducting thin slice $(<1 \mathrm{~mm})$ high-resolution computed tomographic (CT) scans of the mandible with lesions and also scanning of the lower leg, which is the donor site from which the fibular free flap donor is to be harvested. Thereafter, based on the CT scans, tumorous lesions as well as bones are converted into three-dimensional (3D) images, and then resection and reconstruction are planned virtually considering resection margins, osteotomies, and the placement of the fibula bone. The variability of the resection margin is critical in oncologic surgery. Since it is impossible to determine an accurate tumor margin and degree of invasion before an operation, this must be taken into account before proceeding to the second phase.

The second phase is the modeling phase, which uses stereolithographic models. Virtual modeling of the actual surgery is performed. This stage involves preparation of plate templates and cutting guides for both the resection and the vascularized bone graft. What is most important in this phase is to prepare a template for the reconstruction plate that is of sufficient length to protect against the possibility of resecting more than was virtually planned in the first phase, since it is not possible to determine the resection margins.

The third phase is surgery. Performance of actual surgery is done using the cutting guides and plates prepared in the second phase. If the mandibular cancer-free margin is determined in a frozen section, the fibular bone which was almost harvested should be cut according to its defects and then osteomized in situ based on the cutting guide, then fixed to the reconstruction plate. Thereafter, the flap should be inserted and blood flow should be permitted by performing microanastomosis.

The fourth phase is evaluation. This involves performance of high-resolution CT to compare the results with the preoperative virtual plan.

\section{SURGICAL MANAGEMENT ACCORDING TO THE 4 PHASES}

The following case shows how 4 phases of CAS are applied for the segmental mandibular reconstruction after oncological surgery. A 67-year-old-male patient visited our hospital because of multiple skin lesions below his right lower lip that had appeared 2 months prior. He said that the lesions started as small spots, but gradually grew. He received treatment at a local hospital, but the symptoms did not improve. So, he visited our hospital. A biopsy was taken and he was diagnosed with squamous cell carcinoma (Fig. 1A, B). A preoperative tumor workup showed an enhancing mass broadly invading the lip skin and mandible (T4aN0M0). Surgery was planned. Since extensive damage, including damage to the intraoral lining, mandible, and outer skin was expected to occur, double free flap reconstruction using fibular free flap and radial forearm flap was planned. On an enhanced CT scan, the range of the tumor invading the mandible and vascular pedicle where the two flaps were anastomosed was expressed in a $3 \mathrm{D}$ transparent view (Phase I, Fig. 1C, D). To calculate the range of the bone reconstruction, which was the core of the reconstructive surgery, the estimated resection range was expressed in the $3 \mathrm{D}$ model of the tumor-invading mandible (Phase II, Fig. 1E). Then the reconstruction plate was prefabricated as estimated using an actual scale rapidly-prototyped patient mandible template (Phase II, Fig. 1F). A CT angiography image of the lower limb, which was to be the donor site, was taken, and based on this information the donor site was predesignated. On normal CT angiography photos, the axis of the fibular and peroneal artery to be used does not 
match the coronal plane, which may result in incorrect measurement of length. Therefore, the image slicing plane was calibrated to a plane containing axis of the fibular and peroneal artery, and then the length of the donor site, blood vessels, and the site where the bone was to be fractured were measured (Phase II, Fig. 1G, shown in 3D model). Based on this, the flap was designed for surgery (Phase III, Fig. 1H), and the surgeon's expected view during surgery was constructed with 3D images. This enabled the sur- geon to identify the location of the pedicle during surgery, aiding in safe flap harvest (Phase III, Fig. 1I). 3D CT imaging taken 4 weeks after reconstruction showed excellent restoration of the mandibular contour, and continuity was achieved as designed prior to surgery (Phase IV, Fig. 1J, K). Fig. 1L, M shows a photo of the patient taken 6 months after reconstruction, showing that the mandible contour was well-maintained in the absence of drooling.
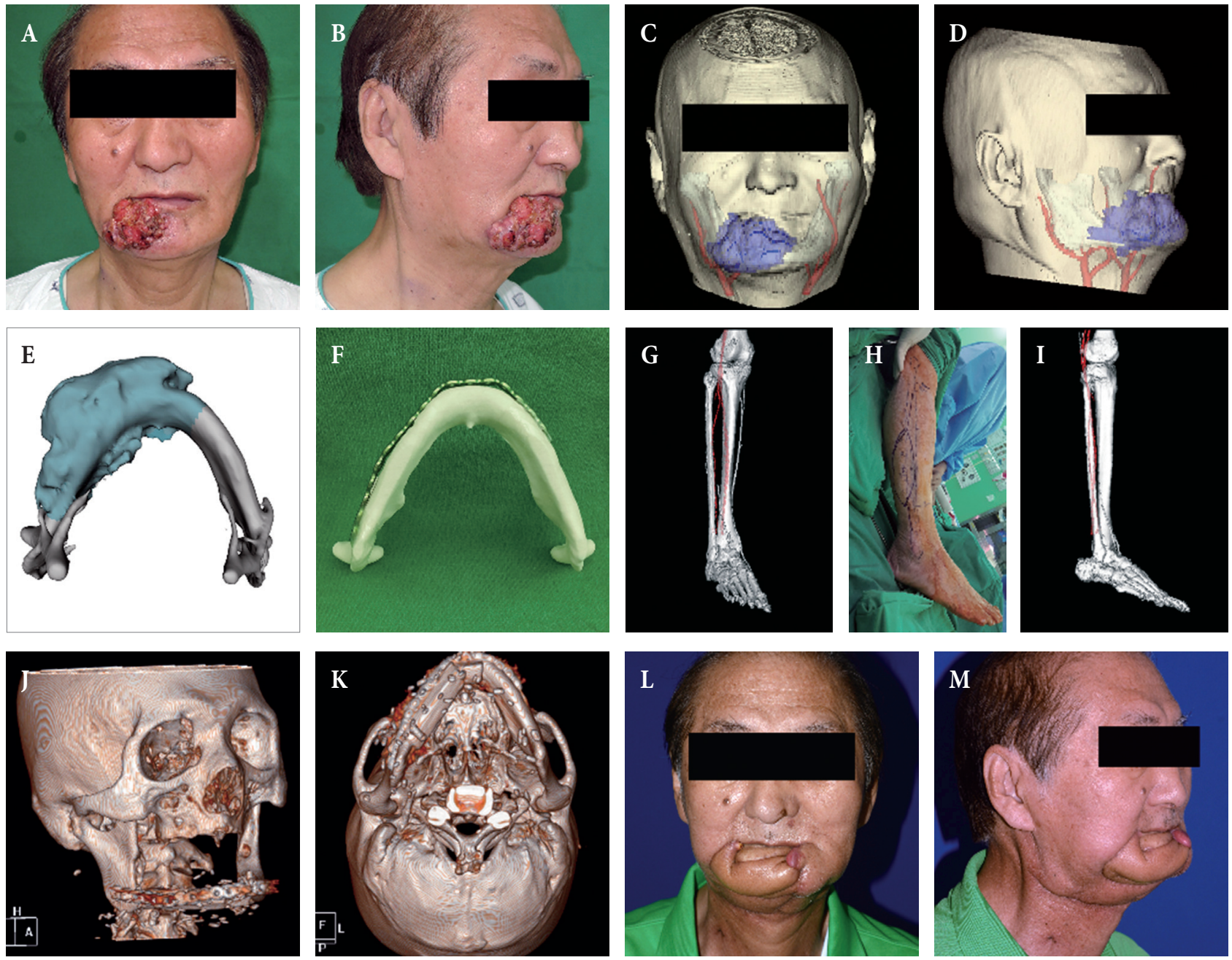

Fig. 1. (A, B) Computer-aided reconstruction of an extensive composite mandibular defect. A 67-year-old male patient with invasive squamous cell carcinoma on the right lower lip. (C, D) A transparent view of the tumor invading the mandibular bone with the vascular pedicle candidates for reconstruction. (E) The tumor-invading mandible was reconstructed as a three-dimensional (3D) object and the resection extent was estimated (dark cyan). (F) Given this estimation, an actual scale rapidly-prototyped patient mandible was manufactured for prefabrication of the reconstruction plate in order to shorten the operation time. (G) For precise preoperative planning and measurement of length, the image slicing plane was calibrated to a plane containing the axis of the donor fibular bone and peroneal artery, and the images were reconstructed as a 3D object. (H) Intraoperative markings and (I) the surgeon's view of the pedicle while harvesting the fibular flap. (J, K) A postoperative 3D view taken four weeks after mandibular reconstruction showing excellent restoration of the mandibular contour and continuity. (L, M) Six-month follow-up view of the patient after adjuvant radiation therapy. 


\section{THE ADVANTAGES OF COMPUTER- AIDED SURGERY}

Because mandibular reconstruction using CAS makes it possible to plan and perform mock surgery in advance, it entails the advantage of being able to increase reconstruction accuracy. When using traditional methods, it is necessary to undergo a lot of trial and error and invest a lot of time in precision work, including alignment of the bending angle of the reconstruction plate $[15,17]$. Since CAS enables the performance of this task with a stereolithographic model, it presents a great advantage in that it is able to reduce ischemia time and operating time $[16,18]$.

According to the results of a literature review, the accuracy of intraoperative planning and in situ plate bending reached $92 \%$ $[7,19]$, and it was effective in increasing the accuracy of proper dental occlusion and the orthognathic relationship $[2,8]$. Furthermore, for surgeons, there is the noted advantage of a reduction in the surgical learning curve [15].

In respect to patients, the cost of surgery increases temporarily because of the need to create a stereolithographic model using an extra plate, but the cost of the treatment as a whole is reduced because of the reduction in entire operation time [18].

\section{LIMITATIONS OF COMPUTER-AIDED SURGERY}

The most important limitation of CAS is that it is unable to determine the tumor margin. For this reason, planning surgery using CAS may require many changes in the operating room. Therefore, the accuracy of a cutting guide is well below the accuracy of other head and neck reconstructive surgical techniques. It is necessary to consider the possibility that more tissue will be resected than planned in the operating room. There are reports that the likelihood of achieving a negative resection margin when performing tumor surgery using CAS is only $5 \%[8,15,20]$.

There are some reports in which, to obtain accuracy of a tumor margin prior to an operation, magnetic resonance imaging was conducted for soft tissue evaluation, and then the results were merged with high-resolution CT scans and CAS was used for tumor resection $[21,22]$. A negative margin was achieved all in three cases, but the results lack reliability because the number of patients to whom this method was applied was very small.

Even though recipient vessels, pedicle length and reach, oral lining and skin deficiency, location and type of skin vessels supplying the osteocutaneous fibula flap skin paddle, and volume restoration/augmentation in mandible reconstruction are crucial elements [23-27], it is difficult to consider and apply these variables in phases II and III of CAS $[9,10,15,16,28]$.

Also, if CAS is used, it is necessary to spend more time preparing for an operation, However, in cases of tumor surgery, surgery within a few days or 1 week is recommended as a tumor continues to progress, but, realistically, this time frame is not easy to maintain. So, patients are asked to undergo surgery within a maximum of 3 weeks, which may also be difficult depending on the situation [29].

\section{AN INTEGRATED PLAN OF COMPUTER-AIDED SURGERY FOR HEAD AND NECK CANCER}

Deek and Wei [14] sharply criticize segmental reconstruction using CAS. They argue that presurgical planning using CAS under the present circumstances is unable to determine the tumor margins and is ineffective considering the effort necessary and that a number of variables that are not considered in CAS can be sufficiently overcome through the experience of well-trained surgeons, which may lead to better results.

We partially agree with them, but occasionally young surgeons rather than experienced surgeons perform reconstruction. In this case, CAS is very effective in reducing the learning curve.

As previously mentioned, CAS will be able to considerably reduce the amount of trial and error necessary in the operative field through the prebending of the reconstruction plate or the identification of an approximate osteotomy site.

Disadvantages of CAS can be overcome by developing new paradigms, such as merging with magnetic resonance images $[21,22]$ and supplementary evidence. In addition, it is challenging 
to set a presurgery preparation period within a few days by simplifying phases I and II.

\section{FUTURE OF COMPUTER-AIDED SURGERY FOR HEAD AND NECK CANCER}

The future direction for CAS is mainly reconstruction using patient-specific 3D printed implants. The fibula bone donor site is a very good donor site, but donor site morbidity is an issue and the microsurgery technique adds to the surgical burden.

In fact, patient-specific 3D printed implants are already being applied to the pelvis, spine, etc. [30-34] Patient-specific three-dimensional printed implants require only the insertion of an implant, so there are no concerns about donor site morbidity, the operation is relatively easy, and the operation time can be reduced.

Studies on patient-specific 3D printed implants in head and neck reconstruction are in progress, but as of yet no systematic reports have been published. This is because there is no substance that can act as a bone substitute in the head and neck. However, in in vivo animal tests, studies using polycaprolactone/beta-tricalcium phosphate are actively in progress $[35,36]$. If these problems are resolved, it will lead to another paradigm shift.

\section{CONCLUSION}

Segmental reconstruction of the mandible using CAS after oncological resection proceeds through 4 phases: planning, modeling, surgery, and evaluation. This helps overcome a lot of trial and error which generally occurs in the operating room and thus reduce operation time. However, it is difficult to predetermine the resection margin at this phase because presurgical recognition of precise tumor margins is required before tumor surgery. However, when considering the variability in the resection margin, CAS is useful in preparing the prebending plate and planning the osteotomy site, which is of great help. Therefore, mandibular reconstruction using CAS is very effective and if the current issues are resolved, the scope of its applications is expected to be greatly extended.

\section{PATIENT CONSENT}

The patient provided written informed consent for the publication and the use of their images.

\section{REFERENCES}

1. Hou JS, Chen M, Pan CB, Tao Q, Wang JG, Wang C, et al. Immediate reconstruction of bilateral mandible defects: management based on computer-aided design/computer-aided manufacturing rapid prototyping technology in combination with vascularized fibular osteomyocutaneous flap. J Oral Maxillofac Surg 2011;69:1792-7.

2. Hayden RE, Mullin DP, Patel AK. Reconstruction of the segmental mandibular defect: current state of the art. Curr Opin Otolaryngol Head Neck Surg 2012;20:231-6.

3. Cordeiro PG, Disa JJ, Hidalgo DA, Hu QY. Reconstruction of the mandible with osseous free flaps: a 10-year experience with 150 consecutive patients. Plast Reconstr Surg 1999;104:1314-20.

4. Hidalgo DA, Pusic AL. Free-flap mandibular reconstruction: a 10year follow-up study. Plast Reconstr Surg 2002;110:438-49.

5. He Y, Zhang ZY, Zhu HG, Sader R, He J, Kovacs AF. Free fibula osteocutaneous flap for primary reconstruction of T3-T4 gingival carcinoma. J Craniofac Surg 2010;21:301-5.

6. Wallace CG, Chang YM, Tsai CY, Wei FC. Harnessing the potential of the free fibula osteoseptocutaneous flap in mandible reconstruction. Plast Reconstr Surg 2010;125:305-14.

7. Bell RB, Weimer KA, Dierks EJ, Buehler M, Lubek JE. Computer planning and intraoperative navigation for palatomaxillary and mandibular reconstruction with fibular free flaps. J Oral Maxillofac Surg 2011;69:724-32.

8. Ciocca L, Mazzoni S, Fantini M, Persiani F, Baldissara P, Marchetti C, et al. A CAD/CAM-prototyped anatomical condylar prosthesis connected to a custom-made bone plate to support a fibula free flap. Med Biol Eng Comput 2012;50:743-9.

9. Hirsch DL, Garfein ES, Christensen AM, Weimer KA, Saddeh PB, Levine JP. Use of computer-aided design and computer-aided manufacturing to produce orthognathically ideal surgical outcomes: a paradigm shift in head and neck reconstruction. J Oral Maxillofac Surg 2009;67:2115-22.

10. Sharaf B, Levine JP, Hirsch DL, Bastidas JA, Schiff BA, Garfein ES. Importance of computer-aided design and manufacturing technology in the multidisciplinary approach to head and neck reconstruction. J Craniofac Surg 2010;21:1277-80.

11. Tepper OM, Sorice S, Hershman GN, Saadeh P, Levine JP, Hirsch D. Use of virtual 3-dimensional surgery in post-traumatic craniomaxillofacial reconstruction. J Oral Maxillofac Surg 2011;69:733-41.

12. Tepper O, Hirsch D, Levine J, Garfein E. The new age of three-dimensional virtual surgical planning in reconstructive plastic surgery. Plast Reconstr Surg 2012;130:192e-194e. 
13. Avraham T, Franco P, Brecht LE, Ceradini DJ, Saadeh PB, Hirsch DL, et al. Functional outcomes of virtually planned free fibula flap reconstruction of the mandible. Plast Reconstr Surg 2014;134:628e-634e.

14. Deek NF, Wei FC. Computer-assisted surgery for segmental mandibular reconstruction with the osteoseptocutaneous fibula flap: can we instigate ideological and technological reforms? Plast Reconstr Surg 2016;137:963-70.

15. Antony AK, Chen WF, Kolokythas A, Weimer KA, Cohen MN. Use of virtual surgery and stereolithography-guided osteotomy for mandibular reconstruction with the free fibula. Plast Reconstr Surg 2011;128:1080-4.

16. Rodby KA, Turin S, Jacobs RJ, Cruz JF, Hassid VJ, Kolokythas A, et al. Advances in oncologic head and neck reconstruction: systematic review and future considerations of virtual surgical planning and computer aided design/computer aided modeling. J Plast Reconstr Aesthet Surg 2014;67:1171-85.

17. Modabber A, Legros C, Rana M, Gerressen M, Riediger D, Ghassemi A. Evaluation of computer-assisted jaw reconstruction with free vascularized fibular flap compared to conventional surgery: a clinical pilot study. Int J Med Robot 2012;8:215-20.

18. Toto JM, Chang EI, Agag R, Devarajan K, Patel SA, Topham NS. Improved operative efficiency of free fibula flap mandible reconstruction with patient-specific, computer-guided preoperative planning. Head Neck 2015;37:1660-4.

19. Patel A, Otterburn D, Saadeh P, Levine J, Hirsch DL. 3D volume assessment techniques and computer-aided design and manufacturing for preoperative fabrication of implants in head and neck reconstruction. Facial Plast Surg Clin North Am 2011;19:683-709.

20. Liu XJ, Gui L, Mao C, Peng X, Yu GY. Applying computer techniques in maxillofacial reconstruction using a fibula flap: a messenger and an evaluation method. J Craniofac Surg 2009;20:372-7.

21. Kraeima J, Schepers RH, van Ooijen PM, Steenbakkers RJ, Roodenburg JL, Witjes MJ. Integration of oncologic margins in three-dimensional virtual planning for head and neck surgery, including a validation of the software pathway. J Craniomaxillofac Surg 2015;43:1374-9.

22. Daisne JF, Duprez T, Weynand B, Lonneux M, Hamoir M, Reychler H, et al. Tumor volume in pharyngolaryngeal squamous cell carcinoma: comparison at CT, MR imaging, and FDG PET and validation with surgical specimen. Radiology 2004;233:93-100.

23. Thoma A, Levis C, Young JE. Oromandibular reconstruction after cancer resection. Clin Plast Surg 2005;32:361-75.

24. Heffelfinger RN, Malhotra PS, Fishman MA. Aesthetic considerations in mandibular reconstruction. Facial Plast Surg 2008;24:35-42.
25. Bak M, Jacobson AS, Buchbinder D, Urken ML. Contemporary reconstruction of the mandible. Oral Oncol 2010;46:71-6.

26. Wei FC, Demirkan F, Chen HC, Chen IH. Double free flaps in reconstruction of extensive composite mandibular defects in head and neck cancer. Plast Reconstr Surg 1999;103:39-47.

27. Wei FC, Celik N, Chen HC, Cheng MH, Huang WC. Combined anterolateral thigh flap and vascularized fibula osteoseptocutaneous flap in reconstruction of extensive composite mandibular defects. Plast Reconstr Surg 2002;109:45-52.

28. Levine JP, Bae JS, Soares M, Brecht LE, Saadeh PB, Ceradini DJ, et al. Jaw in a day: total maxillofacial reconstruction using digital technology. Plast Reconstr Surg 2013;131:1386-91

29. Sink J, Hamlar D, Kademani D, Khariwala SS. Computer-aided stereolithography for presurgical planning in fibula free tissue reconstruction of the mandible. J Reconstr Microsurg 2012;28:395-403.

30. Castilho M, Dias M, Vorndran E, Gbureck U, Fernandes P, Pires I, et al. Application of a 3D printed customized implant for canine cruciate ligament treatment by tibial tuberosity advancement. Biofabrication 2014;6:025005

31. Sutradhar A, Park J, Carrau D, Miller MJ. Experimental validation of $3 \mathrm{D}$ printed patient-specific implants using digital image correlation and finite element analysis. Comput Biol Med 2014;52:8-17.

32. Wong KC, Kumta SM, Geel NV, Demol J. One-step reconstruction with a 3D-printed, biomechanically evaluated custom implant after complex pelvic tumor resection. Comput Aided Surg 2015;20:14-23.

33. Xu N, Wei F, Liu X, Jiang L, Cai H, Li Z, et al. Reconstruction of the Upper Cervical Spine Using a Personalized 3D-Printed Vertebral Body in an Adolescent With Ewing Sarcoma. Spine (Phila Pa 1976) 2016;41:E50-4.

34. Chen X, Xu L, Wang Y, Hao Y, Wang L. Image-guided installation of 3D-printed patient-specific implant and its application in pelvic tumor resection and reconstruction surgery. Comput Methods Programs Biomed 2016;125:66-78.

35. Shim JH, Huh JB, Park JY, Jeon YC, Kang SS, Kim JY, et al. Fabrication of blended polycaprolactone/poly(lactic-co-glycolic acid)/beta-tricalcium phosphate thin membrane using solid freeform fabrication technology for guided bone regeneration. Tissue Eng Part A 2013;19:317-28.

36. Shim JH, Yoon MC, Jeong CM, Jang J, Jeong SI, Cho DW, et al. Efficacy of rhBMP-2 loaded PCL/PLGA/beta-TCP guided bone regeneration membrane fabricated by $3 \mathrm{D}$ printing technology for reconstruction of calvaria defects in rabbit. Biomed Mater 2014;9:065006. 